\title{
Synthesis of novel fluorophenylaryl / heteroaryl ether derivatives
}

\author{
Biplab K. Das, ${ }^{\text {a }}$ Norio Shibata, ${ }^{b}$ Kazuyuki Harada, ${ }^{\dagger}$ and Takeshi Toru ${ }^{b}$ \\ ${ }^{a}$ Department of Pharmacy, East West University, 43 Mohakhali C/A, Dhaka-1212, Bangladesh \\ ${ }^{b}$ Department of Applied Chemistry, Graduate School of Engineering, Nagoya Institute of \\ Technology, Gokiso-cho, Showa-ku, Nagoya 463-0031, Japan \\ E-mail: bkdas72@yahoo.com
}

\begin{abstract}
A detailed syntheses of some fluorophenylaryl / heteroaryl ether derivatives 1a, $\mathbf{b}-\mathbf{4 a}$, b have been described which was accomplished by using a variety of convenient phenolic coupling methods and subsequent $\mathrm{Pd}-\mathrm{C}$ catalyzed hydrogenation of the coupled intermediates. The compounds synthesized are structurally similar to a number of anti-inflammatory agents.
\end{abstract}

Keywords: Synthesis, flurophenylaryl / heteroaryl, ether derivatives, phenolic coupling, hydrogenation

\section{Introduction}

Earlier we published a research article where we described briefly the existence of intramolecular C-F---H-N hydrogen bonding using compounds 1-4, which were designed as covalently-linked base pair models of $\mathbf{F}$ (difluorotoluene deoxynucleoside) and $\mathbf{A}$ (adenine deoxynucleoside) (Figure 1). ${ }^{1,2}$ Although our study failed to find any C-F---H-N intramolecular hydrogen bonding and supported Kool's theory that shape complementarities between the base pairs play the major role in DNA replication fidelity, ${ }^{3}$ several papers have been published subsequently showing intramolecular C-F--H-N interactions and the role of organic fluorine in hydrogen bonding by means of density functional theory, ab initio and MMFF force field calculations. ${ }^{4}$ We couldn't explain definitely the reason of not finding intramolecular C-F---H-N hydrogen bonding and it was assumed that the problem might be the design of the compounds. Therefore we didn't proceed for further investigation. Later on we found that our compounds are structurally similar to a number of biologically interesting fluorophenylaryl ether type compounds (Figure 2). ${ }^{5,6,7}$ The most promising compounds belonging this class is Flosulide (I) and its sulfone analogue (II), which have already been proven as selective cyclo-oxygenase-2

\footnotetext{
${ }^{\dagger}$ Faculty of Pharmaceutical Sciences, Toyama Medical and Pharmaceutical, University, Sugitani 2630, Toyama 930-0194, Japan
} 
(COX-2) inhibitors ${ }^{6,7}$. Besides these, 5'-cyano-2'(2,4-difluorophenoxy)-biphenyl-4-sulfonamide (III), 2-benzyl-6-cyclohexylsulfanyl-5-(3-fluoro-phenoxy)-1H-benzimidazole (IV) have antiinflammatory properties ${ }^{8,9}$ and 4-(biphenyl-4-yloxy)-3-fluorobenzoic acid (V) is a potent human prostatic 5 - $\alpha$-reductase inhibitor. ${ }^{10}$ Therefore these compounds are therapeutically important as analgesic, antipyretic, antiarthritic (especially rheumatoid arthritis) and anticancer agents. The compounds 1-4 are expected to have similar biological activities due to their structural similarities with these compounds (Figure 2). In this paper we disclose the detailed synthetic procedures with characterization of these compounds. These compounds can be utilized as the lead compound for the design of some biologically active compounds.

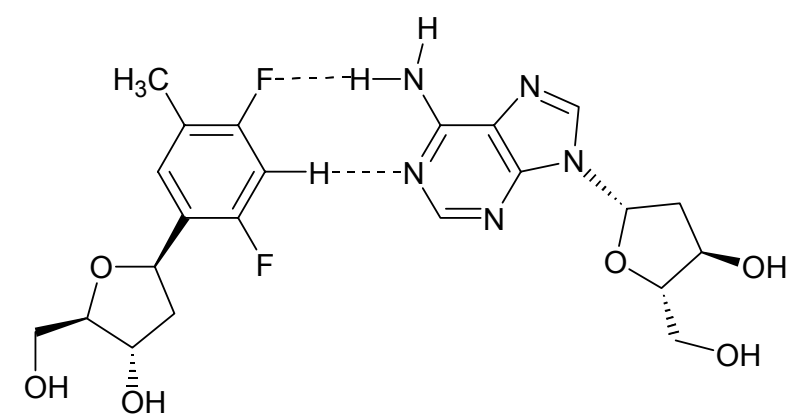

F (Difluorotoluene deoxynucleoside)
A (Adenine deoxynucleoside)<smiles>[X]c1ccc(Oc2ccc3[nH]cnc3c2N)c([X])c1</smiles><smiles>[X]c1ccc(Oc2ccccc2N)c([X])c1</smiles><smiles>[X]c1ccc(Oc2cccnc2N)c([X])c1</smiles>

1a $X=F, Y=H$

1b $X=H, Y=F$

2a $X=F, Y=H$

2b $X=H, Y=F$

3a $X=F, Y=H$

3b $X=H, Y=F$

4a $X=F, Y=H$

4b $X=H, Y=F$

\section{Figure 1}<smiles>COS(=O)(=O)c1cc2c(cc1Oc1ccc(F)cc1F)CCC2=O</smiles>

Flosulide (I)<smiles>COS(=O)(=O)Nc1cc2c(cc1Oc1ccc(F)cc1F)CCO2</smiles>

II<smiles>N#Cc1ccc(Oc2ccc(F)cc2F)c(-c2ccc(S(N)(=O)=O)cc2)c1</smiles>

III<smiles>Fc1cccc(Oc2cc3nc(Cc4ccccc4)[nH]c3cc2SC2CCCCC2)c1</smiles>

IV<smiles>O=C(O)c1ccc(Oc2ccc(-c3ccccc3)cc2)c(F)c1</smiles>

$\mathrm{V}$

Figure 2. Some reported biologically active fluorophenylaryl / heteroaryl ether compounds. 


\section{Results and Discussion}

The synthesis of compounds 1-4 was accomplished by using a variety of phenol coupling methods as key steps (Scheme 1 and 2). 4-Fluoro-1,2-phenylenediamine $\mathbf{5}^{11}$ was first converted into 5-fluorobenzimidazole $\mathbf{6}$ by heating in formic acid solution. Nitration of $\mathbf{6}$ under relatively mild conditions gave a mixture of two regioisomers 5-fluoro-4-nitrobenzimidazole 7 and 5fluoro-6-nitrobenzimidazole 8 . The 4-nitro compound 7 was subjected to $\mathrm{KF}^{-} \mathrm{Al}_{2} \mathrm{O}_{3}{ }^{12}$ mediated coupling with $o$-fluorophenol and $p$-fluorophenol in the presence of catalytic amount of 18crown-6-ether ${ }^{13}$ in $\mathrm{MeCN}$ to give fluorophenyl benzimidazole (heteroaryl) ethers 9a-9b. Pd-C catalyzed hydrogenation of $\mathbf{9 a}-\mathbf{9 b}$ at atmospheric pressure afforded compounds $\mathbf{1 a}-\mathbf{1} \mathbf{b}$, respectively in excellent yields.

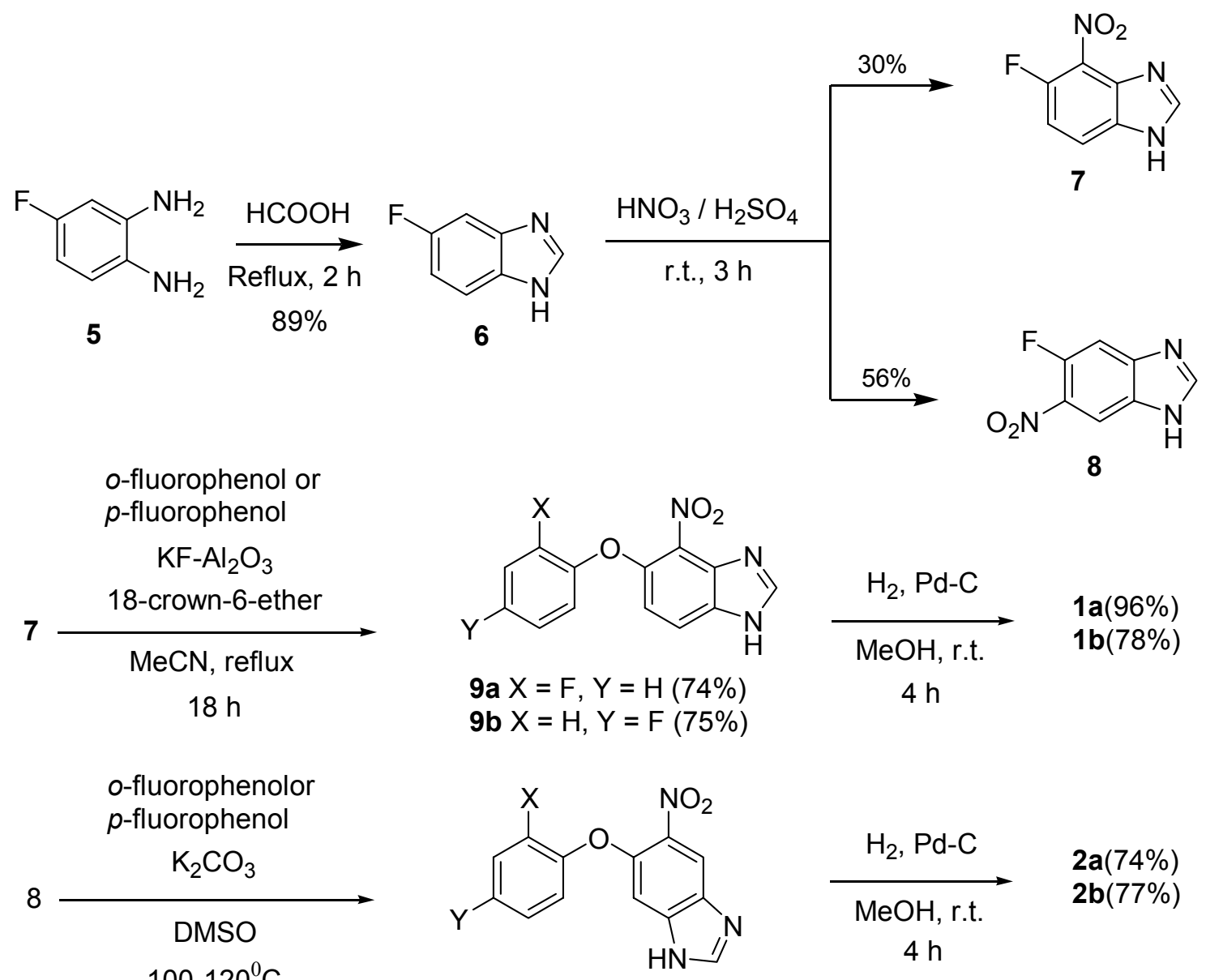

10a $X=F, Y=H(13 \%)$

$10 \mathrm{~b} X=H, Y=F(12 \%)$

\section{Scheme 1}

However, $\mathrm{KF}-\mathrm{Al}_{2} \mathrm{O}_{3}$ mediated coupling reaction ${ }^{12,13}$ of 6-nitro compound 8 with fluorophenols failed unexpectedly. We then investigated an alternative procedure. The coupling 
reaction of 8 with $o$-fluorophenol and $p$-fluorophenol was performed in the presence of $\mathrm{K}_{2} \mathrm{CO}_{3}$ in DMSO at $100-120^{\circ} \mathrm{C}^{10 \mathrm{a}}$ affording the fluorophenyl benzimidazole (heteroaryl) ethers $\mathbf{1 0 a}-\mathbf{1 0 b}$, which on hydrogenation gave the desired compounds $\mathbf{2 a - 2 b}$, respectively in good yields.

Preparation of the model compounds 3 and $\mathbf{4}$ required different phenolic coupling conditions. Coupling of commercially available 2-chloronitrobenzene 11 with the potassium salt of $o$ fluorophenol at $110-120{ }^{\circ} \mathrm{C}^{5 \mathrm{~b}}$ yielded the fluorophenylaryl ether compound 12a which on Pd-C catalyzed hydrogenation gave 3a. The compound $\mathbf{4 a}$ was prepared from $\mathrm{NaH}$ based coupling ${ }^{14}$ of $13^{15}$ with $o$-fluorophenol to afford the fluorophenyl phenyl pyridyl (heteroaryl) ether 14a with subsequent $\mathrm{Pd} / \mathrm{C}$ catalyzed hydrogenation. $p$-Fluorinated compounds $\mathbf{3 b}$ and $\mathbf{4 b}$ were prepared in similar sequences from 12 and 14, respectively in good yields (Scheme 2).
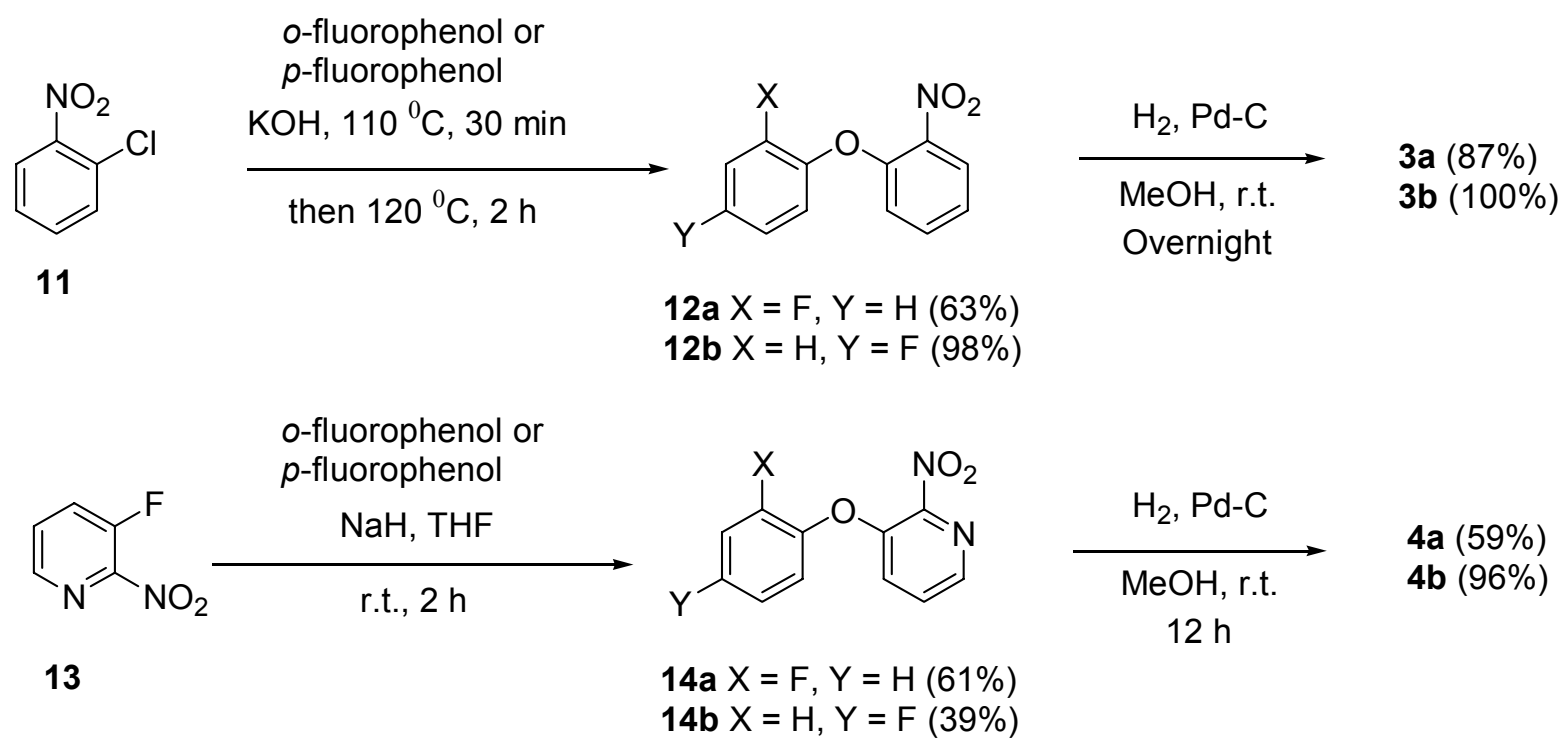

\section{Scheme 2}

In summary, we have demonstrated the detailed syntheses of some novel fluorophenylaryl / heteroaryl ether derivatives which are structurally similar to some potent anti-inflammatory agents like Flosulide. The synthetic procedures are more straightforward and convenient than the traditional multi-step syntheses. These synthetic studies are expected to be helpful to generate some highly potent compounds of similar biological activities. The biological activity studies of the synthesized compounds are in progress.

\section{Experimental Section}

General Procedures. All melting points were determined with a Yanagimoto Micro Melting Point apparatus and are uncorrected. IR spectra $\left(\mathrm{cm}^{-1}\right)$ were recorded on a Perkin-Elmer 1600 spectrometer. ${ }^{1} \mathrm{H}-\mathrm{NMR}$ spectra were measured as solutions in $\mathrm{CDCl}_{3}, \mathrm{CD}_{3} \mathrm{OD}, \mathrm{D}_{2} \mathrm{O}$ or DMSO-$d_{6}$ 
and chemical shifts are expressed in ppm relative to internal $\mathrm{Me}_{4} \mathrm{Si}(0.00 \mathrm{ppm})$ and were recorded on a JEOL GX-270 (270 MHz) spectrometer. ${ }^{19} \mathrm{~F}$ - NMR spectra were measured with $\mathrm{CFCl}_{3}$ as an internal standard and were taken with a JEOL GX-270 (254 MHz) spectrometer. Upfield shifts were quoted as negative $\delta$ values. ${ }^{13} \mathrm{C}$ NMR spectra were recorded at 125.76 , 75.46 and $68 \mathrm{MHz}$ using Unity plus 500, Varian Gemini 300 and JEOL GX-270 instruments. Chemical shifts are quoted in ppm and are referenced to $\mathrm{CDCl}_{3}$. Electron ionization (EI) mass spectra were taken with a JEOL JMS-D300 spectrometer. Column chromatography and preparative TLC were performed on BW-200 (Fuji Silysia) and Kieselgel 60 (Merck, art. 7748), respectively. All reactions were carried out under a dry $\mathrm{N}_{2}$ atmosphere. Unless otherwise noted, reagents were added by syringe. $\mathrm{MeOH}$ was distilled from $\mathrm{CaO}$ and $\mathrm{DMF}$ was distilled over $\mathrm{CaH}_{2}$ immediately prior to use. Commercially available dehydrated THF [stabilized with butylated hydroxytoluene (BHT)] was used for reaction.

5-Fluorobenzimidazole (6). A solution of 4-fluoro-1, 2-phenylenediamine 5 (3.5 g, $27.7 \mathrm{mmol})$ in $90 \%$ formic acid $(50 \mathrm{ml})$ was heated at reflux for $2 \mathrm{~h}$. Following removal of the solvent, the residue was chromatographed on silica gel [chloroform- methanol (9:1)] to yield 7 as a yellowish solid (3.36 g, 89\%) which was recrystallized from chloroform; mp 87-88 ${ }^{\circ} \mathrm{C}$; IR $\left(\mathrm{KBr}, \mathrm{cm}^{-1}\right.$ ) 3108, 3062, 1597, 1335; ${ }^{1} \mathrm{H}-\mathrm{NMR}\left(\mathrm{CDCl}_{3}\right)$ 7.04-7.62 (3H, m, ArH), $8.22(1 \mathrm{H}$, br s, $\mathrm{N}=\mathrm{CH}-\mathrm{NH})$; ${ }^{19} \mathrm{~F}-\mathrm{NMR}\left(\mathrm{CDCl}_{3}\right)-118.9(\mathrm{~m})$; MS m/z (EI) $136\left(\mathrm{M}^{+}\right), 135\left(\mathrm{M}^{+}-1\right)$; HRMS Calcd. $\mathrm{C}_{7} \mathrm{H}_{5} \mathrm{~N}_{2} \mathrm{~F}$ : 136.0437, Found: 136.0440 .

5-Fluoro-4-nitrobenzimidazole (7) and 5-Fluoro-6-nitrobenzimidazole (8). To a solution of $6(5.5 \mathrm{~g}, 40.4 \mathrm{mmol})$ in concentrated sulphuric acid $(6.4 \mathrm{ml}, 120 \mathrm{mmol})$ was added concentrated nitric acid $(5.1 \mathrm{ml}, 120 \mathrm{mmol})$ slowly and the whole mixture was stirred at room temperature for 3h. The solution was poured into ice-water. A solid was formed which was filtered and subsequently washed with water and saturated sodium bicarbonate solution. The liquid portion was neutralized by adding $\mathrm{KOH}$ pellets. The solid still present was filtered off and the mother liquid was extracted with ethyl acetate $(100 \mathrm{ml} \times 2)$. The total solids were taken and the organic portion was concentrated in vacuo. The residue was chromatographed on silica gel (3-5\% methanol in dichloromethane) to give $7(2.2 \mathrm{~g}, 30 \%)$ and 8 (4.07 g, 56\%), both as a yellowish solid. Data for 7: mp 191-192 ${ }^{\circ} \mathrm{C}$ (from dichloromethane); ${ }^{1} \mathrm{H}-\mathrm{NMR}\left(5 \% \mathrm{CD}_{3} \mathrm{OD}\right.$ in $\mathrm{CDCl}_{3}$ ) $7.25(1 \mathrm{H}, \mathrm{dd}, J=2.7,8.1 \mathrm{~Hz}, \mathrm{ArH}), 8.09(1 \mathrm{H}, \mathrm{dd}, J=3.7,8.7 \mathrm{~Hz}, \mathrm{ArH}), 8.50(1 \mathrm{H}$, br s, N=CH$\mathrm{NH}) ;{ }^{19} \mathrm{~F}-\mathrm{NMR}\left(5 \% \mathrm{CD}_{3} \mathrm{OD}\right.$ in $\left.\mathrm{CDCl}_{3}\right)-120.5(\mathrm{~m}) ; \mathrm{MS} m / z(\mathrm{EI}) 181\left(\mathrm{M}^{+}\right), 135\left(\mathrm{M}^{+}-\mathrm{NO}_{2}\right)$; HRMS Calcd. $\mathrm{C}_{7} \mathrm{H}_{4} \mathrm{O}_{2} \mathrm{~N}_{3} \mathrm{~F}$ : 181.0296, Found: 181.0297. Data for 8: mp $189-190{ }^{\circ} \mathrm{C}$ (from dichloromethane); IR (KBr, cm ${ }^{-1}$ ) 3601, 3107, 2981, 1532, 1423, 1304; ${ }^{1} \mathrm{H}-\mathrm{NMR}\left(5 \% \mathrm{CD}_{3} \mathrm{OD}\right.$ in $\left.\mathrm{CDCl}_{3}\right) 7.50(1 \mathrm{H}, \mathrm{d}, J=10.9 \mathrm{~Hz}, \mathrm{ArH}), 8.35(1 \mathrm{H}$, br s, N=CH-NH$), 8.45(1 \mathrm{H}, \mathrm{d}, J=6.3 \mathrm{~Hz}$, ArH); ${ }^{19} \mathrm{~F}-\mathrm{NMR}\left(5 \% \mathrm{CD}_{3} \mathrm{OD}\right.$ in $\left.\mathrm{CDCl}_{3}\right)-124.0(\mathrm{~m})$; MS $m / z(\mathrm{EI}) 181\left(\mathrm{M}^{+}\right), 182\left(\mathrm{M}^{+}+1\right), 135$ $\left(\mathrm{M}^{+}-\mathrm{NO}_{2}\right)$; HRMS Calcd. $\mathrm{C}_{7} \mathrm{H}_{4} \mathrm{O}_{2} \mathrm{~N}_{3} \mathrm{~F}$ : 181.0305, Found: 181.0307 . 
5-(2-Fluorophenyl)-4-nitrobenzimidazole ether (9a). To a stirred solution of 7 (150 mg, 0.83 $\mathrm{mmol})$ in a mixture of acetonitrile $(10 \mathrm{ml})$ and DMSO $(0.5 \mathrm{ml})$ were added 18-crown-6-ether (219 mg, $0.25 \mathrm{mmol}), \mathrm{KF}-\mathrm{Al}_{2} \mathrm{O}_{3}(700 \mathrm{mg}), o$-fluorophenol $(78.0 \mathrm{mg}, 0.69 \mathrm{mmol})$ and refluxed at $100{ }^{\circ} \mathrm{C}$ for $18 \mathrm{~h}$. The reaction mixture was first filtered and to the filtrate $0.5 \mathrm{M} \mathrm{KOH}(10 \mathrm{ml})$ and ethyl acetate $(100 \mathrm{ml})$ were added. The layers were separated and the organic layer was washed with brine, dried over anhydrous $\mathrm{Na}_{2} \mathrm{SO}_{4}$ and concentrated in vacuo. The residue was chromatographed on silica gel (dichloromethane-methanol 9:1) to yield the product 9a (166 mg, $74 \%$ ) as a brown crystalline solid; mp $162-163{ }^{\circ} \mathrm{C}$ (from dichloromethane); Anal. Calcd. $\mathrm{C}_{13} \mathrm{H}_{8} \mathrm{~N}_{3} \mathrm{O}_{3} \mathrm{~F}$ : C, 57.15; N, 15.38, H, 2.95, Found: C, 56.90; N, 15.37; H, 2.99; IR (KBr, cm $\left.{ }^{-1}\right)$ 2992, 1580, 1538, 1341, 1033, 765; ${ }^{1} \mathrm{H}-\mathrm{NMR}\left(\mathrm{CDCl}_{3}\right)$ 6.91-7.26 (5H, m, ArH + benzimidazole 6-H), $8.01(1 \mathrm{H}, \mathrm{d}, J=8.7 \mathrm{~Hz}$, benzimidazole $7-H), 8.24\left(1 \mathrm{H}\right.$, br s, N=CH-NH); ${ }^{19} \mathrm{~F}-\mathrm{NMR}$ $\left(\mathrm{CDCl}_{3}\right)-130.5(\mathrm{~m}) ; \mathrm{MS} m / z(\mathrm{EI}) 273\left(\mathrm{M}^{+}\right), 274\left(\mathrm{M}^{+}+1\right)$; HRMS Calcd. $\mathrm{C}_{13} \mathrm{H}_{8} \mathrm{~N}_{3} \mathrm{O}_{3} \mathrm{~F}$ : 273.0550, Found: 273.0571.

5-(4-Fluorophenyl)-4-nitrobenzimidazole ether (9b). Compound 9b (223 mg, 75\%) was prepared from the coupling of $p$-fluorophenol $(123 \mathrm{mg}, 1.10 \mathrm{mmol})$ with 8 (200 $\mathrm{mg}, 1.10 \mathrm{mmol})$ by the procedure analogous to $9 \mathbf{a}$ as a brown solid; mp $198-200{ }^{\circ} \mathrm{C}$ (from dichloromethane); Anal. Calcd. $\mathrm{C}_{13} \mathrm{H}_{8} \mathrm{~N}_{3} \mathrm{O}_{3} \mathrm{~F}$ : C, 57.15; N, 15.38, H, 2.95, Found: C, 57.12; N, 15.44; H, 2.84; IR $\left(\mathrm{KBr}, \mathrm{cm}^{-1}\right)$ 2998, 1579, 1539, 1343, 1091, 782; ${ }^{1} \mathrm{H}-\mathrm{NMR}\left(\mathrm{CDCl}_{3}\right)$ 6.94-7.24 (5H, m, ArH + benzimidazole 6-H), $8.03(1 \mathrm{H}, \mathrm{m}$, benzimidazole $7-H), 8.22(1 \mathrm{H}, \mathrm{d}, J=8.9 \mathrm{~Hz}, \mathrm{~N}=\mathrm{CH}-\mathrm{NH})$; ${ }^{19} \mathrm{~F}-\mathrm{NMR}\left(\mathrm{CDCl}_{3}\right)-118.9(\mathrm{~m})$; MS $m / z(\mathrm{EI}) 273\left(\mathrm{M}^{+}\right), 274\left(\mathrm{M}^{+}+1\right), 227\left(\mathrm{M}^{+}-\mathrm{NO}_{2}\right)$; HRMS Calcd. $\mathrm{C}_{13} \mathrm{H}_{8} \mathrm{~N}_{3} \mathrm{O}_{3} \mathrm{~F}: 273.0550$, Found: 273.0531 .

5-(2-Fluorophenyl)-4-aminobenzimidazole ether (1a). To a solution of 9a (107 mg, 0.391 $\mathrm{mmol})$ in $\mathrm{MeOH}(10 \mathrm{ml})$ under $\mathrm{N}_{2}$ was added $\mathrm{Pd} / \mathrm{C}(20 \mathrm{mg})$ and $\mathrm{H}_{2}$ gas was allowed to pass through the solution with stirring for $4 \mathrm{~h}$ until completion of the reaction by TLC. The reaction mixture was filtered off through a plug of celite and the filtrate was concentrated in vacuo. The residue was purified by silica gel column chromatography (chloroform-methanol 9:1) to yield 1a (91 mg, 96\%) as a grey crystalline solid; mp $127-128{ }^{\circ} \mathrm{C}$ (from dichloromethane); Anal. Calcd. $\mathrm{C}_{13} \mathrm{H}_{10} \mathrm{~N}_{3} \mathrm{OF}$ : C, 64.19; N, 17.28, H, 4.14, Found: C, 63.98; N, 17.42; H, 4.15; IR (KBr, cm $\left.{ }^{-1}\right)$ 3439, $3358\left(\mathrm{NH}_{2}\right), 3156,3055,1500,1368,1252,797 ;{ }^{1} \mathrm{H}-\mathrm{NMR}$ (DMSO-d $)_{6} 5.08(2 \mathrm{H}$, br s, $\left.\mathrm{NH}_{2}\right), 6.65-7.29(6 \mathrm{H}, \mathrm{m}, \mathrm{ArH}+$ benzimidazole- $H), 8.10(1 \mathrm{H}$, br s, $\mathrm{N}=\mathrm{CH}-\mathrm{NH})$; ${ }^{13} \mathrm{C}-\mathrm{NMR}$ $\left(\mathrm{CD}_{3} \mathrm{OD}\right) 102.74,117.57,118.82,123.71,123.80,125.47,125.53,130.63,134.02,137.25$, 141.63, 147.61, 154.27; ${ }^{19}$ F-NMR (DMSO- $\left.d_{6}\right)-133.9(\mathrm{~m})$; MS $m / z$ (EI) $243\left(\mathrm{M}^{+}\right), 223\left(\mathrm{M}^{+}{ }_{-}\right.$ HF); HRMS Calcd. $\mathrm{C}_{13} \mathrm{H}_{10} \mathrm{~N}_{3} \mathrm{OF}$ : 243.0808, Found: 243.0782 .

5-(4-Fluorophenyl)-4-aminobenzimidazole ether (1b). Compound 1 b (76.0 mg, 78\%) was prepared by $\mathrm{Pd} / \mathrm{C}(30.0 \mathrm{mg})$ catalyzed reduction of $1 \mathrm{a}(109 \mathrm{mg}, 0.399 \mathrm{mmol})$ as a grey crystalline solid; mp $186-188{ }^{\circ} \mathrm{C}$ (from dichloromethane); Anal. Calcd. $\mathrm{C}_{13} \mathrm{H}_{10} \mathrm{~N}_{3} \mathrm{OF}$ : C, 64.19; $\mathrm{N}, 17.28$, H, 4.14, Found: C, 64.12; N, 17.30; H, 4.12; IR (KBr, cm $\left.{ }^{-1}\right) 3386\left(\mathrm{NH}_{2}\right), 3156,2912$, 1628, 1503, 964; ${ }^{1} \mathrm{H}-\mathrm{NMR}\left(\mathrm{DMSO}-d_{6}\right) 4.99\left(2 \mathrm{H}\right.$, br s, $\left.\mathrm{NH}_{2}\right), 6.75-7.15(6 \mathrm{H}, \mathrm{m}, \operatorname{ArH}+$ benzimidazole- $H), 8.09$ (1H, br s, $\mathrm{N}=\mathrm{CH}-\mathrm{NH}) ;{ }^{19} \mathrm{~F}-\mathrm{NMR}\left(\mathrm{DMSO}-d_{6}\right)-122.5$ (m); MS $m / z \quad$ (EI) $243\left(\mathrm{M}^{+}\right), 244\left(\mathrm{M}^{+}+1\right), 242\left(\mathrm{M}^{+}-1\right)$; HRMS Calcd. $\mathrm{C}_{13} \mathrm{H}_{10} \mathrm{~N}_{3} \mathrm{OF}: 243.0808$, Found: 243.0785. 
5-(2-Fluorophenyl)-6-nitrobenzimidazole ether (10a). To a stirred solution of $\mathbf{8}$ (200 $\mathrm{mg}, 1.10$ mmol) in DMSO $(5 \mathrm{ml})$ were added $o$-fluorophenol $(0.147 \mathrm{ml}, 1.10 \mathrm{mmol})$ and anhydrous $\mathrm{K}_{2} \mathrm{CO}_{3}(334 \mathrm{mg}, 2.20 \mathrm{mmol})$ and the mixture was heated at $100{ }^{\circ} \mathrm{C}$ for one week. The reaction mixture was diluted with ethyl acetate $(100 \mathrm{ml})$ and was washed successively with $1 \mathrm{M} \mathrm{KOH} \mathrm{(10}$ $\mathrm{ml})$, water $(20 \mathrm{ml})$, and brine $(20 \mathrm{ml})$. The organic layer dried over anhydrous $\mathrm{Na}_{2} \mathrm{SO}_{4}$, concentrated in vacuo and the residue was purified by column chromatography over silica gel (chloroform-methanol 9:1) to yield the product $\mathbf{1 0 a}(38.0 \mathrm{mg}, 13 \%)$ as a brown crystalline solid; $\mathrm{mp} 216-217{ }^{\circ} \mathrm{C}$ (from chloroform); IR $\left(\mathrm{KBr}, \mathrm{cm}^{-1}\right.$ ) 3100, 2963, 1588, 1530, 1333, 740; ${ }^{1} \mathrm{H}-\mathrm{NMR}$ $\left(\mathrm{CD}_{3} \mathrm{OD}\right) 7.03-7.30(5 \mathrm{H}, \mathrm{m}, \mathrm{ArH}+$ benzimidazole $4-H), 8.33(1 \mathrm{H}$, br s, $\mathrm{N}=\mathrm{CH}-\mathrm{NH}), 8.39(1 \mathrm{H}$, br s, benzimidazole 7-H); ${ }^{19} \mathrm{~F}-\mathrm{NMR}\left(\mathrm{CD}_{3} \mathrm{OD}\right)-131.9(\mathrm{~m})$; $\mathrm{MS} m / z(\mathrm{EI}) 273\left(\mathrm{M}^{+}\right), 272\left(\mathrm{M}^{+}-1\right)$; HRMS Calcd. $\mathrm{C}_{13} \mathrm{H}_{8} \mathrm{~N}_{3} \mathrm{O}_{3} \mathrm{~F}: 273.0559$, Found: 273.0549

5-(4-Fluorophenyl)-6-nitrobenzimidazole ether (10b). Compound 10b (35.0 mg, 12\%) was prepared from the coupling of $p$-fluorophenol (134 mg, $1.10 \mathrm{mmol})$ with 8 (200 $\mathrm{mg}, 1.10 \mathrm{mmol})$ by the procedure analogous to $\mathbf{1 0 a}$ as a brown crystalline solid; mp $181-185{ }^{\circ} \mathrm{C}$ (from chloroform); Anal. Calcd. $\mathrm{C}_{13} \mathrm{H}_{8} \mathrm{~N}_{3} \mathrm{O}_{3} \mathrm{~F}$ : C, 57.15; N, 15.38, H, 2.95, Found: C, 57.06; N, 15.41; $\mathrm{H}, 3.04$.); IR (KBr, cm ${ }^{-1}$ ) 3102, 1533, 1500, 1336, 1278, 755; 1H-NMR (CD $\left.{ }_{3} \mathrm{OD}\right)$ 6.99-7.15 $(4 \mathrm{H}, \mathrm{m}, \mathrm{ArH}), 7.27(1 \mathrm{H}$, br s, benzimidazole $4-H), 8.31(1 \mathrm{H}$, br s, $\mathrm{N}=\mathrm{CH}-\mathrm{NH}), 8.38(1 \mathrm{H}$, br s, benzimidazole 7-H); ${ }^{19} \mathrm{~F}-\mathrm{NMR}\left(\mathrm{CD}_{3} \mathrm{OD}\right)-120.2$ (m); MS $m / z$ (EI) $273\left(\mathrm{M}^{+}\right), 272\left(\mathrm{M}^{+}-1\right)$; HRMS Calcd. $\mathrm{C}_{13} \mathrm{H}_{8} \mathrm{~N}_{3} \mathrm{O}_{3} \mathrm{~F}: 273.0550$, Found: 273.0558 .

5-(2-Fluorophenyl)-6-aminobenzimidazole ether (2a). To the solution of 10a (20.0 mg, 0.073 $\mathrm{mmol})$ in dry methanol $(10 \mathrm{ml})$ was added $\mathrm{Pd} / \mathrm{C}(10 \mathrm{mg})$ and $\mathrm{H}_{2}$ gas was allowed to pass for $4 \mathrm{~h}$. The reaction mixture was filtered through a plug of celite and the filtrate was concentrated in vacuo to give the residue which was purified by preparative TLC (chloroform-methanol 9:1) to yield 2a (13.0 mg, 74\%) as a grey crystalline solid; $\mathrm{mp} 99-100{ }^{\circ} \mathrm{C}$ (from chloroform-methanol); IR $\left(\mathrm{KBr}, \mathrm{cm}^{-1}\right) 3374,3122\left(\mathrm{NH}_{2}\right), 2960,1500,1364,1259,750 ;{ }^{1} \mathrm{H}-\mathrm{NMR}$ (DMSO- $\left.d_{6}\right) 4.79(2 \mathrm{H}$, br s, $\left.\mathrm{NH}_{2}\right), 6.85-7.34(6 \mathrm{H}, \mathrm{m}, \mathrm{ArH}+$ benzimidazole- $H), 8.26(1 \mathrm{H}$, br s, $\mathrm{N}=\mathrm{C} H-\mathrm{NH})$; ${ }^{19} \mathrm{~F}-\mathrm{NMR}$ $\left(\mathrm{DMSO}-d_{6}\right)-132.8(\mathrm{~m})$; MS $m / z(\mathrm{EI}) 243\left(\mathrm{M}^{+}\right), 244\left(\mathrm{M}^{+}+1\right), 223\left(\mathrm{M}^{+}-\mathrm{HF}\right)$; HRMS Calcd. $\mathrm{C}_{13} \mathrm{H}_{10} \mathrm{~N}_{3} \mathrm{OF}$ : 243.0808, Found: 243.0831.

5-(4-Fluorophenyl)-6-aminobenzimidazole ether (2b). Compound $\mathbf{2 b}(10.0 \mathrm{mg}$, 75\%) was prepared by Pd-C (10 mg) catalyzed reduction of $\mathbf{1 0 b}(15 \mathrm{mg}, 0.054 \mathrm{mmol})$ as a grey crystalline solid; mp 208-209 ${ }^{\circ} \mathrm{C}$ (from chloroform-methanol); IR ( $\left.\mathrm{KBr}\right) / \mathrm{cm}^{-1} 3787,3431\left(\mathrm{NH}_{2}\right), 1501$, 1416, 1362, 759; ${ }^{1} \mathrm{H}-\mathrm{NMR}\left(\mathrm{DMSO}-d_{6}\right) 4.72\left(2 \mathrm{H}\right.$, br s, $\left.\mathrm{NH}_{2}\right), 6.92-7.16(6 \mathrm{H}, \mathrm{m}, \mathrm{ArH}+$ benzimidazole- $H$ ), $7.92(1 \mathrm{H}$, br s, $\mathrm{N}=\mathrm{C} H-\mathrm{NH}) ;{ }^{19} \mathrm{~F}-\mathrm{NMR}\left(\mathrm{DMSO}-d_{6}\right)-119.2(\mathrm{~m}) ; \mathrm{MS} m / z$ (EI) $243\left(\mathrm{M}^{+}\right), 244\left(\mathrm{M}^{+}+1\right), 227\left(\mathrm{M}^{+}-\mathrm{NH}_{2}\right)$; HRMS Calcd. $\mathrm{C}_{13} \mathrm{H}_{10} \mathrm{~N}_{3} \mathrm{OF}$ : 243.0807, Found $\mathrm{M}^{+}$ 243.080 .

1-(2-Fluorophenyl)-2-nitrobenzene ether (12a). To a stirred mixture of $o$-fluorophenol (1.00 g, $8.92 \mathrm{mmol})$ and dried powdered $\mathrm{KOH}(500 \mathrm{mg}, 8.92 \mathrm{mmol})$ heated at $110^{\circ} \mathrm{C}$ for 30 minutes was added 2-chloronitrobenzene $11(1.40 \mathrm{~g}, 8.92 \mathrm{mmol})$ in one portion and heated at $120^{\circ} \mathrm{C}$ for $2 \mathrm{~h}$. The reaction mixture was diluted with ethyl acetate $(100 \mathrm{ml})$ and brine $(20 \mathrm{ml})$ was added to it. The whole mixture was successively washed with $1 \mathrm{M} \mathrm{NaOH}(20 \mathrm{ml}), 1 \mathrm{M} \mathrm{HCl}(20 \mathrm{ml})$ and 
brine $(20 \mathrm{ml})$. The organic portion was dried over anhydrous $\mathrm{Na}_{2} \mathrm{SO}_{4}$ and concentrated in vacuo. The residue was chromatographed on silica gel (hexane-ethyl acetate 9:1) to give 12a (1.31 g, $63 \%$ ) as a yellow oil; IR (neat, $\left.\mathrm{cm}^{-1}\right) 3091,1584,1529,1356 ;{ }^{1} \mathrm{H}-\mathrm{NMR}\left(\mathrm{CDCl}_{3}\right) 6.91-7.23(6 \mathrm{H}$, m, FAr-H), 7.48-7.52 (1H, m, NO $2-A r H), 7.98$ (1H, dd, $\left.J=1.3,8.3 \mathrm{~Hz}, \mathrm{NO}_{2}-\mathrm{ArH}\right)$; ${ }^{19} \mathrm{~F}-\mathrm{NMR}$ $\left(\mathrm{CDCl}_{3}\right)-130.4(\mathrm{~m})$; MS m/z (EI) $233\left(\mathrm{M}^{+}\right), 234\left(\mathrm{M}^{+}+1\right)$; HRMS Calcd. $\mathrm{C}_{12} \mathrm{H}_{8} \mathrm{NO}_{3} \mathrm{~F}$ : 233.0488, Found: 233.050 .

1-(4-Fluorophenyl)-2-nitrobenzene ether (12b). Compound 12b (2.03 g, 98\%) was prepared by following the procedure analogous to 12a using 11 (1.68 g, $10.7 \mathrm{mmol}), p$-fluorophenol (1.0 $\mathrm{g}, 8.92 \mathrm{mmol}$ ) and $\mathrm{KOH}\left(600 \mathrm{mg}, 10.7 \mathrm{mmol}\right.$ ) as a yellow oil; IR (neat, $\mathrm{cm}^{-1}$ ) 3079, 2871, 1529 , 1353; ${ }^{1} \mathrm{H}-\mathrm{NMR}\left(\mathrm{CDCl}_{3}\right)$ 6.95-7.19 (6H, m, $\left.\mathrm{ArH}+\mathrm{NO}_{2}-\mathrm{ArH}\right), 7.51$ (1H, m, NO $\left.\mathrm{N}_{2}-\mathrm{ArH}\right), 7.94$ $\left(1 \mathrm{H}, \mathrm{dd}, J=1.3,8.3 \mathrm{~Hz}, \mathrm{NO}_{2}-\mathrm{ArH}\right) ;{ }^{19} \mathrm{~F}-\mathrm{NMR}\left(\mathrm{CDCl}_{3}\right)-118.6(\mathrm{~m}) ; \mathrm{m} / z(\mathrm{EI}) 233\left(\mathrm{M}^{+}\right), 234\left(\mathrm{M}^{+}+\right.$ 1), $187\left(\mathrm{M}^{+}-\mathrm{NO}_{2}\right)$; HRMS Calcd. $\mathrm{C}_{12} \mathrm{H}_{8} \mathrm{O}_{3} \mathrm{NF}$ : 233.0472, Found: 233.0434.

2-(2-Fluorophenyl) phenylamine ether (3a). To a solution of 12a (200 $\mathrm{mg}, 0.858 \mathrm{mmol})$ in anhydrous methanol $(10 \mathrm{ml})$ under nitrogen was added $\mathrm{Pd} / \mathrm{C}(50.0 \mathrm{mg})$. The flask was evacuated and hydrogen gas was allowed to pass through the solution overnight. After being ensured of the completion of the reaction by TLC, the reaction mixture was filtered through a plug of celite to remove $\mathrm{Pd} / \mathrm{C}$ and the filtrate was concentrated and purified by column chromatography on silica gel (hexane-ethyl acetate 9:1) to give 3a $\left(151 \mathrm{mg}, 87 \%\right.$ ) as a yellow oil; IR (neat, $\mathrm{cm}^{-1}$ ) 3364 , $3333\left(\mathrm{NH}_{2}\right), 3038,2868,1308,782 ;{ }^{1} \mathrm{H}-\mathrm{NMR}\left(\mathrm{CDCl}_{3}\right) 3.79\left(2 \mathrm{H}\right.$, br s, $\left.\mathrm{NH}_{2}\right), 6.65-6.83(4 \mathrm{H}, \mathrm{m}$, $\left.\mathrm{NH}_{2}-\mathrm{ArH}\right), 6.96-7.18$ (4H, m, F-ArH); ${ }^{19} \mathrm{~F}-\mathrm{NMR}\left(\mathrm{CDCl}_{3}\right)-133.2(\mathrm{~m}) ; \mathrm{MS} \mathrm{m} / \mathrm{z}$ (EI) $203\left(\mathrm{M}^{+}\right)$, $204\left(\mathrm{M}^{+}+1\right), 187\left(\mathrm{M}^{+}-\mathrm{NH}_{2}\right)$; HRMS Calcd. $\mathrm{C}_{12} \mathrm{H}_{10} \mathrm{ONF}$ : 203.0746, Found: 203.0721.

2-(4-Fluorophenyl) phenylamine ether (3b). Compound 3b (355 $\mathrm{mg}, 100 \%)$ was prepared by the $\mathrm{Pd} / \mathrm{C}(100 \mathrm{mg})$ catalyzed reduction of $\mathbf{1 2 b}(400 \mathrm{mg}, 1.72 \mathrm{mmol})$ as a yellow oil; IR (neat, $\left.\mathrm{cm}^{-1}\right)$ 3471, $3383\left(\mathrm{NH}_{2}\right), 3002,1269,1201,703 ;{ }^{1} \mathrm{H}-\mathrm{NMR}\left(\mathrm{CDCl}_{3}\right) 3.79\left(2 \mathrm{H}, \mathrm{br} \mathrm{s}, \mathrm{NH}_{2}\right), 6.66-$ 6.89 (4H, m, NH$-\mathrm{ArH}), 6.94-7.02$ (4H, m, F-ArH); ${ }^{19} \mathrm{~F}-\mathrm{NMR}\left(\mathrm{CDCl}_{3}\right)-121.7$ (m); MS m/z (EI) $203\left(\mathrm{M}^{+}\right), 204\left(\mathrm{M}^{+}+1\right), 187\left(\mathrm{M}^{+}-\mathrm{NH}_{2}\right)$; HRMS Calcd. $\mathrm{C}_{12} \mathrm{H}_{10} \mathrm{ONF}$ : 203.0768, Found: 203.0746 .

3-(2-Fluorophenyl)-2-nitropyridyl ether (14a). To a stirred dispersion of $o$-fluorophenol $(0.32$ $\mathrm{ml}, 3.52 \mathrm{mmol})$ and $\mathrm{NaH}(140 \mathrm{mg}, 5.83 \mathrm{mmol})$ in THF $(5 \mathrm{ml})$ was added 3-fluoro-2nitropyridine $13(100 \mathrm{mg}, 0.703 \mathrm{mmol})$ and stirred for $2 \mathrm{~h}$. The reaction was quenched by saturated aqueous $\mathrm{NH}_{4} \mathrm{Cl}(30 \mathrm{ml})$ solution and extracted with ethyl acetate $(250 \mathrm{ml})$. The organic layer was washed with brine $(100 \mathrm{ml})$, dried over $\mathrm{Na}_{2} \mathrm{SO}_{4}$, filtered and concentrated in vacuo. The crude residue was purified by column chromatography over silica gel (hexanedichloromethane $6: 4)$ to give $\mathbf{1 4 a}\left(101 \mathrm{mg}, 61 \%\right.$ ) as a yellow oil; IR (neat, $\mathrm{cm}^{-1}$ ) 1545, 1368 $\left(\mathrm{NO}_{2}\right) ;{ }^{1} \mathrm{H}-\mathrm{NMR}\left(\mathrm{CDCl}_{3}\right) 6.86(1 \mathrm{H}, \mathrm{m}, \mathrm{ArH}), 6.96-7.09(3 \mathrm{H}, \mathrm{m}, \mathrm{ArH}), 7.20-7.35(2 \mathrm{H}, \mathrm{m}$, Pyridyl- $H), 8.24(1 \mathrm{H}$, br d, $J 4.3, \mathrm{NCH}=\mathrm{CH}) ;{ }^{19} \mathrm{~F}-\mathrm{NMR}\left(\mathrm{CDCl}_{3}\right)-141.5(\mathrm{~m}) ; \mathrm{MS} \mathrm{m} / z$ (EI) 234 $\left(\mathrm{M}^{+}\right), 235\left(\mathrm{M}^{+}+1\right)$; HRMS Calcd. $\mathrm{C}_{11} \mathrm{H}_{7} \mathrm{~N}_{2} \mathrm{O}_{3} \mathrm{~F}$ : 234.0441, Found: 234.0439.

3-(4-Fluorophenyl)-2-nitropyridyl ether (14b). Compound 14b (128 mg, 39\%) was prepared by following the procedure analogous to $\mathbf{1 4 a}$ using 13 (200 $\mathrm{mg}, 1.41 \mathrm{mmol}), p$-fluorophenol (789 $\mathrm{mg}, 7.04 \mathrm{mmol}$ ) and $\mathrm{NaH}\left(282 \mathrm{mg}, 7.04 \mathrm{mmol}\right.$ ) as a yellowish solid; $\mathrm{mp} 61-62{ }^{\circ} \mathrm{C}$ (from hexane- 
ethyl acetate); Anal. Calcd. $\mathrm{C}_{11} \mathrm{H}_{7} \mathrm{~N}_{2} \mathrm{O}_{3} \mathrm{~F}$ : C, 56.42; N, 11.96; H, 3.01, Found: C, 56.28; N, 11.81; H, 3.31; IR (KBr, cm $\left.{ }^{-1}\right)$ 1547, $1372\left(\mathrm{NO}_{2}\right)$; ${ }^{1} \mathrm{H}-\mathrm{NMR}\left(\mathrm{CDCl}_{3}\right)$ 7.07-7.15 (4H, m, F-ArH), 7.37 $(1 \mathrm{H}, \mathrm{m}$, Pyridyl- $H), 7.49(1 \mathrm{H}, \mathrm{m}$, Pyridyl- $H), 8.24(1 \mathrm{H}$, br d, $J 3.2, \mathrm{NCH}=\mathrm{CH}) ;{ }^{19} \mathrm{~F}-\mathrm{NMR}$ $\left(\mathrm{CDCl}_{3}\right)-116.9(\mathrm{~m})$; MS m/z (EI) $234\left(\mathrm{M}^{+}\right), 235\left(\mathrm{M}^{+}+1\right)$; HRMS Calcd. $\mathrm{C}_{11} \mathrm{H}_{7} \mathrm{~N}_{2} \mathrm{O} 3 \mathrm{~F}: 234.044$, Found: 234.0439.

3-(2-Fluorophenyl)-2-pyridylamine ether (4a). To the solution of 14a (100 $\mathrm{mg}, 0.427 \mathrm{mmol})$ in methanol $(5 \mathrm{ml})$ was added $\mathrm{Pd} / \mathrm{C}(50 \mathrm{mg})$ and hydrogen gas was passed through the solution for $12 \mathrm{hr}$. until the completion of the reaction. The reaction mixture was filtered through a plug of celite. The filtrate was concentrated in vacuo and purified by preparative TLC (ethyl acetate $100 \%)$ to give $4 \mathbf{a}(51 \mathrm{mg}, 58 \%)$ as a yellow oil; IR (neat, $\left.\mathrm{cm}^{-1}\right)$ 3481, $3305\left(\mathrm{NH}_{2}\right), 3146,2927$, 1481, 1251; ${ }^{1} \mathrm{H}-\mathrm{NMR}\left(\mathrm{DMSO}-d_{6}\right) 5.99$ (2H, br s, $\left.\mathrm{NH}_{2}\right), 6.49-7.07$ (3H, m, ArH), 7.18-7.39 (3H, m, $\mathrm{Ar} H+$ Pyridyl- $H), 7.75\left(1 \mathrm{H}\right.$, br d, $\left.J=4.3 \mathrm{~Hz}, \mathrm{NH}_{2} \mathrm{C}=\mathrm{NCH}\right) ;{ }^{19} \mathrm{~F}-\mathrm{NMR}\left(\mathrm{CDCl}_{3}\right)-131.7(\mathrm{~m})$; MS m/z (EI) $204\left(\mathrm{M}^{+}\right), 188\left(\mathrm{M}^{+}-\mathrm{NH}_{2}\right)$; HRMS Calcd. $\mathrm{C}_{11} \mathrm{H}_{9} \mathrm{~N}_{2} \mathrm{OF}: 204.0699$, Found: 204.0677. 3-(4-Fluorophenyl)-2-pyridylamine ether (4b). Compound 4b (83.0 mg, 96\%)was prepared from Pd-C (100 mg) catalyzed reduction of $\mathbf{1 4 b}(100 \mathrm{mg}, 0.427 \mathrm{mmol})$ according to the procedure analogous to 4a, as an oil; IR (neat, $\left.\mathrm{cm}^{-1}\right)$ 3471, $3283\left(\mathrm{NH}_{2}\right), 3002,1508,1269,1144$, 753; ${ }^{1} \mathrm{H}-\mathrm{NMR}$ (DMSO- $\left.d_{6}\right) 5.89$ (2H, br s, $\left.\mathrm{NH}_{2}\right), 6.51-7.01(4 \mathrm{H}, \mathrm{m}, \mathrm{ArH}+$ Pyridyl-H), 7.15-7.22 $(2 \mathrm{H}, \mathrm{m}$, Pyridyl- $H), 7.75\left(1 \mathrm{H}\right.$, br d, $\left.J 4.3, \mathrm{NH}_{2} \mathrm{C}=\mathrm{NCH}\right) ;{ }^{19} \mathrm{~F}-\mathrm{NMR}\left(\mathrm{CDCl}_{3}\right)-119.9(\mathrm{~m})$; $\mathrm{MS} \mathrm{m} / z$ (EI) $204\left(\mathrm{M}^{+}\right), 188\left(\mathrm{M}^{+}-\mathrm{NH}_{2}\right)$; HRMS Calcd. $\mathrm{C}_{11} \mathrm{H}_{9} \mathrm{~N}_{2} \mathrm{OF}$ : 204.0699, Found: 204.0761.

\section{Acknowledgements}

This work was partially supported by a Grant-in-aid from the Ministry of Education, Science, Sports and Culture, Japan. N.S. wishes to thank the Kato Memorial Bioscience Foundation for support. We thank Prof. Yoshio Takeuchi (Faculty of Pharmaceutical Sciences, Toyama Medical and Pharmaceutical University, Japan) very much for generously sharing his facilities, kind encouragement and for giving us the opportunity to study in the field of fluorine chemistry.

\section{References and Notes}

1. Shibata, N.; Das, B. K.; Harada, K.; Takeuchi, Y.; Bando, M. Synlett 2001, 1755.

2. (a) Qiao, X.; Kishi, Y. Angew. Chem. Int. Ed. 1999, 38, 928. (b) Qiu, Y.-L.; Li, H.-Y.; Topalov, G.; Kishi, Y. Tetrahedron Lett. 2000, 41, 9425. (c) Li, H.-Y.; Qiu, Y.-L.; Moyroud, E.; Kishi, Y. Angew. Chem. Int. Ed. 2001, 40, 1471.

3. (a) Schweitzer, B. A.; Kool, E. T. J. Am. Chem. Soc. 1995, 117, 1863. (b) Guckian, K. M.; Kool, E. T. Angew. Chem. Int. Ed. 1997, 36, 2825. (c) Kool, E. T. Acc. Chem. Res. 2002, 35, 936. (d) O’Neill, B. M.; Ratto, J. E.; Good, K. L.; Tahmassebi, D. C.; Helquist, S. A.; Morales, J. C.; Kool, E. T. J. Org. Chem. 2002, 67, 5869. (e) Kool, E. T. Biopolymers 1998, 
48, 3. (f) Diederichsen, U. Angew. Chem. Int. Ed. 1998, 37, 1655. (g) Morales, J. C.; Kool, E. T. Nat. Struct. Biol. 1998, 5, 950. (h) Guckian, K. M.; Krugh, T. R.; Kool, E. T. Nat. Struct. Biol. 1998, 5, 954. (i) Barsky, D.; Kool E. T.; Colvin, M. E. J. Biomol. Struct. Dynamics 1999, 16, 1119.

4. (a) Paulini, R.; Muller, K.; Diederich, F. Angew. Chem. Int. Ed. 2005, 44, 1788. (b) Chowdhury, R.; Urs, U. K.; Guru Row, T. N.; Nagarajan, K. J. Mol. Struc. 2002, 605(1), 71. (c) Haufe, G.; Rosen, T. C.; Meyer, O. G. J.; Frohlich, R.; Rissanen, K. J. Fluorine Chem. 2002, 114, 189. (d) Lankin, D. C.; Hardcastle, K.; Snyder, J. P. Chem. Eur. J. 2005, 11, 1579 .

5. (a) Klein, T.; Nusing, R. M.; Pfeilschifter, J.; Ullrich, V. Biochem. Pharmacol. 1994, 48, 1605. (b) Li, C-H.; Soucy-Breau, C.; Ouimet, N. Synthesis 1995, 1355. (c) Cameron, B. W.; Benoit, G. Synthesis 1998, 8, 1101.

6. (a) Hisaki, C.; Hiroshi, K.; Shinji, M.; Jun, Nitta; Keiichi, T.; Takihiro, I. PCT Int. Appl. 1996, 141pp. (b) Shunatro, T.; Chosaku, Y.; Takihiro, I.; Keiichi, T.; Ryuko, T.; Hideyoshi, N.; Tomoya, S.; Shinji, M. Ger. Offen. 1989, 142. (c) Clemens, R.; Frank, B.; Eberhard, S.; Irmgard, B.; Schering A.-G, F. Eur. J. Med. Chem. 1982, 17, 173.

7. Dannhardt, G.; Kiefer, W. Eur. J. Med. Chem. 2001, 36, 109.

8. Saito, S.; Matsumoto, T.; Nakmura, T. Jpn. Kokai Tokkyo Koho 2000, 42pp.

9. (a) Goldstein, D. M.; Hawley, R. C.; Lui, A. Sui-ting.; Sjogren, E. B. PCT Int. Appl. 2003, 61pp. (b) Borzilleri, R.M.; Cornelius, Lyndon A. M.; Schmidt, R.J.; Schroeder, G. M.; Kim, K. S. U.S. Pat. Appl. Publ. 2005, 128pp.

10. (a) Igarashi, S.; Kimura, T.; Naito, R.; Hara, H.; Fuji, M.; Koutoku, H.; Oritani, H.; Mase, T. Chem. Pharm. Bull. 1999, 47, 1073. (b) Johnson, W. O.; Kollman, G. E.; Swithenbank, C.; Yih, R. Y. J. Agric. Food Chem. 1978, 26, 285.

11. Kano, S.; Shibuya, S.; Yuasa, Y. J. Heterocycl. Chem. 1980, 17, 1559.

12. Schmittling, E. A.; Sawyer, J. S. Tetrahedron Lett. 1991, 32, 7207.

13. Sawyer, J. S.; Schmittling, E. A.; Palkowitz, J. A.; Smith III, W. J. J. Org. Chem. 1998, 63, 6338.

14. Eicher, T.; Fey, S.; Puhl, W.; Buchel, E.; Speicher, A. Eur. J. Org. Chem. 1988, 877.

15. Ple, N.; Queguiner, G. J. Heterocycl. Chem., 1989, 26, 475. 